\title{
Depletion of a Microtubule-Associated Motor Protein Induces the Loss of Dendritic Identity
}

\author{
Wenqian Yu, ${ }^{1}$ Crist Cook, ${ }^{1}$ Carley Sauter, ${ }^{1}$ Ryoko Kuriyama, ${ }^{2}$ Paul L. Kaplan, ${ }^{3}$ and Peter W. Baas ${ }^{1}$ \\ ${ }^{1}$ Department of Anatomy, The University of Wisconsin Medical School, Madison, Wisconsin 53706, ${ }^{2}$ Department of \\ Genetics, Cell Biology and Development, The University of Minnesota Medical School, Minneapolis, Minnesota 55455, and \\ ${ }^{3}$ Creative BioMolecules, Hopkinton, Massachusetts 01748
}

\begin{abstract}
Dendrites are short stout tapering processes that are rich in ribosomes and Golgi elements, whereas axons are long thin processes of uniform diameter that are deficient in these organelles. It has been hypothesized that the unique morphological and compositional features of axons and dendrites result from their distinct patterns of microtubule polarity orientation. The microtubules within axons are uniformly oriented with their plus ends distal to the cell body, whereas microtubules within dendrites are nonuniformly oriented. The minus-end-distal microtubules are thought to arise via their specific transport into dendrites by the motor protein known as CHO1/MKLP1. According to this model, CHO1/MKLP1 transports microtubules with their minus ends leading into dendrites by generating forces against the plus-end-distal microtubules, thus creating drag on the plusend-distal microtubules. Here we show that depletion of $\mathrm{CHO} 1 /$ MKLP1 from cultured neurons causes a rapid redistribution of
\end{abstract}

microtubules within dendrites such that minus-end-distal microtubules are chased back to the cell body while plus-end-distal microtubules are redistributed forward. The dendrite grows significantly longer and thinner, loses its taper, and acquires a progressively more axon-like organelle composition. These results suggest that the forces generated by CHO1/MKLP1 are necessary for maintaining the minus-end-distal microtubules in the dendrite, for antagonizing the anterograde transport of the plus-end-distal microtubules, and for sustaining a pattern of microtubule organization necessary for the maintenance of dendritic morphology and composition. Thus, we would conclude that dendritic identity is dependent on forces generated by CHO1/MKLP1.

Key words: dendrite; axon; neuron; microtubule; CHO1/ $M K L P 1 ;$ motor protein
Typical vertebrate neurons extend a single axon and several dendrites. Dendrites are different from the axon both morphologically and compositionally. The axon is a long slender process of uniform diameter that is effectively unlimited in its growth potential. In contrast, dendrites are stout tapering processes that remain quite short compared to axons. Axons are deficient in ribosomes and Golgi elements, whereas dendrites are rich in these organelles. The means by which neurons generate and maintain these differences is a central unanswered question in cellular neuroscience. We have proposed that most or all of the features that distinguish dendrites from the axon might be attributable, either directly or indirectly, to differences in the organization of microtubules within these processes (Black and Baas, 1989). Unlike the uniformly plus-end-distal polarity pattern of axonal microtubules, dendritic microtubules have a nonuniform polarity pattern (Baas et al., 1988). During neuronal development, plus-end-distal microtubules arise first within immature processes and are then followed by the gradual addition of minusend-distal microtubules to those processes that become dendrites (Baas et al., 1989). The appearance of the minus-end-distal microtubules corresponds with the acquisition of many distinctive features of dendritic morphology and composition, suggesting a mechanistic link between microtubule organization and the distinct structural and compositional features of these processes. This conclusion is also suggested by experimental studies in which key features of axonal and dendritic morphology were shown to be mimicked by

\footnotetext{
Received March 15, 2000; revised April 20, 2000; accepted May 10, 2000.

This work was funded by grants from the National Institutes of Health and the National Science Foundation to P.W.B. We thank Dennis Higgins, David Sharp, and Itzhak Fischer for helpf ul discussions. We thank Itzhak Fischer and Lester Binder for providing antibodies.

Correspondence should be addressed to Peter W. Baas, Department of Anatomy, The University of Wisconsin Medical School, 1300 University Avenue, Madison, WI 53706. E-mail: pwbaas@facstaff.wisc.edu.

Copyright (C) 2000 Society for Neuroscience $0270-6474 / 00 / 205782-10 \$ 15.00 / 0$
}

non-neuronal cells experimentally induced to extend processes with either uniform or nonuniform microtubule polarity patterns, respectively (Sharp et al., 1996, 1997a).

These observations have led to a model whereby the key event in dendritic differentiation is the specific transport of minus-enddistal microtubules into the dendrite (Sharp et al., 1995; Baas and Yu, 1996; Baas, 1999). Studies from our laboratory strongly suggest that the motor protein known as CHO1/MKLP1 is essential for the appearance of minus-end-distal microtubules within developing neurites and for their differentiation into dendrites (Yu et al., 1997; Sharp et al., 1997b; Ferhat et al., 1998). Studies in vitro have shown that CHO1/MKLP1 transports microtubules with their minus ends leading toward the plus ends of other microtubules and does so by generating forces against the oppositely oriented microtubules (Nislow et al., 1992). We have proposed that plus-end-distal microtubules are transported into axons and dendrites by another motor, cytoplasmic dynein, and that CHO1/MKLP1 generates its forces by pushing against these microtubules (Baas, 1999). According to our model, this would create drag on the anterograde movement of the plus-end-distal microtubules, thus slowing the growth of the dendrite compared to the axon. In addition, profound changes in membrane traffic would result from the establishment of a nonuniform microtubule polarity pattern. These changes would contribute to establishing the unique features that distinguish dendrites from the axon. For example, ribosomes and Golgi elements are thought to move toward minus ends of microtubules, and hence would have an appropriate track to move into dendrites but not the axon (Black and Baas, 1989).

If this reasoning is correct, the identity of the dendrite is dependent on the forces generated by CHO1/MKLP1 on the microtubule array. To test this, we have now performed studies in which we experimentally depleted this motor protein from neurons with well developed dendrites. As a result, we observe a rapid redistribution of microtubules of each orientation and concomitant changes in both the morphology and composition of the dendrite. 


\section{MATERIALS AND METHODS}

Cell culture. Cultures of rat sympathetic neurons were generated from the superior cervical ganglia of newborn rats essentially as previously described (Baas and Ahmad, 1993), except that OP1 was added to the cultures 1 week after plating to assist in the rapid differentiation of robust dendritic arbors in low-density cultures (Sharp et al., 1997b).

Oligonucleotide treatments. As in our previous studies (Yu et al., 1997; Sharp et al., 1997b), translation of CHO1/MKLP1 was suppressed by treatment of cultures with phosphorothioate-substituted DNA oligonucleotides (Research Genetics, Huntsville, AL). The antisense oligonucleotide consisted of the sequence 5'-AGCTTTCGCTGGTTTCATG-3', which is the inverse complement of the coding sequence -1 to +18 of hamster CHO1/MKLP1 transcript (Kuriyama et al., 1994). Oligonucleotides were stored in serum-free medium, aliquoted, and frozen at $-80^{\circ} \mathrm{C}$. Two weeks after plating, by which time robust dendrites had formed, a portion of the plating medium was removed, and new medium containing the oligonucleotides was added at a final concentration of $1 \mu \mathrm{M}$. This medium was changed with fresh oligonucleotide-containing medium every $12 \mathrm{hr}$ for the duration of the experiment. As a negative "sense" control, oligonucleotides with the inverse complement of the antisense sequence were added to dishes in an identical manner as the antisense oligonucleotides.

Immunofluorescence microscopy. Neuronal cultures were immunostained in single-label analyses with one of four different primary antibodies. A mouse monoclonal antibody called RMDO20 (which recognizes a poorly phosphorylated neurofilament epitope) was purchased from Zymed (San Francisco, CA). The mouse monoclonal antibody called CHO1 (which recognizes CHO1/MKLP1) was generated as previously described (Sellitto and Kuriyama, 1988). A mouse monoclonal antibody termed tau-1 (which recognizes a phosphorylated variant of tau) was provided by Lester Binder. A mouse monoclonal antibody that recognizes the full-length variant of microtubule-associated protein-2 (MAP-2) was provided by Itzhak Fischer. In the case of CHO1, this antibody was used at a concentration of 1:1000, and an appropriate Cy3-conjugated secondary antibody (purchased from Jackson ImmunoResearch, West Grove, PA) was used at $1: 1500$. In the case of the RMDO20, the antibody was used at 1:500. The tau-1 and MAP-2 antibodies were used at 1:100. In all cases other than CHO1, the secondary antibody (Cy3-conjugated and purchased from Jackson ImmunoResearch) was used at 1:1000. For CHO1 staining, cultures were rinsed briefly in PBS, fixed for $6 \mathrm{~min}$ in cold methanol $\left(-20^{\circ} \mathrm{C}\right)$, rehydrated three times for $5 \mathrm{~min}$ each in $\mathrm{PBS}$, exposed to a blocking solution (containing 5\% normal goat serum) for $30 \mathrm{~min}$, exposed to the primary antibody overnight at $4^{\circ} \mathrm{C}$, rinsed extensively, exposed to the secondary antibody for $1 \mathrm{hr}$ at $37^{\circ} \mathrm{C}$, rinsed extensively, and then mounted in a medium that reduces photobleaching. For neurofilament staining, the same procedure was used, except that cultures were fixed with a $15 \mathrm{~min}$ exposure to $4 \%$ formaldehyde, and then post-extracted with $0.5 \%$ Triton X-100 for 5 min. For tau-1 or MAP-2 staining, the same procedure was used, except that the cultures were fixed with a $20 \mathrm{~min}$ exposure to a solution containing $4 \%$ paraformaldehyde and $0.1 \%$ glutaraldehyde, postextracted in graded ethanols, and then rehydrated. Photographs of the fluorescent cells were obtained using the LSM 410 confocal microscope (Zeiss, Thornwood, NY) with the pinhole wide open. In the case of the CHO1-stains, all images were taken at identical brightness and contrast settings so that fluorescence quantification could be performed. Levels of fluorescence within individual cells were quantified as previously described (Yu et al., 1997; Sharp et al., 1997b).

Lucifer yellow dye injections. For injection of Lucifer yellow dye into neurons, we used the protocol of Higgins et al. (1991). A quantity of $\sim 4 \mathrm{pl}$ of a solution of $10 \mathrm{mg} / \mathrm{ml}$ Lucifer yellow (Molecular Probes, Eugene, OR) was microinjected into a small number of neurons in each culture. The neurons were spaced sufficiently far apart to ensure little or no overlap in their neuritic arbors. After permitting the dye to diff use throughout the neuron for $1 \mathrm{hr}$, the cultures were fixed in PBS containing 4\% paraformaldehyde. Photographs of the fluorescent cells were obtained using the LSM 410 confocal microscope with the pinhole wide open.

Electron microscopy. For standard electron microscopy, we used previously reported procedures (Yu and Baas, 1994, 1995). Neuron cultures were fixed for $20 \mathrm{~min}$ at $37^{\circ} \mathrm{C}$ in $2 \%$ glutaraldehyde, post-fixed with $1 \%$ $\mathrm{OsO}_{4}$, rinsed twice for $2 \mathrm{~min}$ in $\mathrm{NaCl}$, rinsed twice for $2 \mathrm{~min}$ in water, contrasted for $30 \mathrm{~min}$ in $5 \%$ aqueous uranyl acetate, dehydrated in ethanols, and embedded in LX-112 (Ladd, Burlington, VT). After curing overnight at $60^{\circ} \mathrm{C}$, the glass coverslips were dissolved by exposure to hydrofluoric acid. Cells of interest were circled using a diamond-marker objective, and their phase-contrast images were recorded using a video printer (Sony, Tokyo, Japan). Thin sections of a uniform thickness of 100 nm were obtained with an Ultracut S Ultramicrotome (Reichert-Jeng, Vienna, Austria), stained with uranyl acetate and lead citrate, and observed and photographed with a transmission electron microscope. The precise points along the length of the dendrite corresponding to each electron micrograph were recorded on the video prints.

Microtubule polarity analyses. To determine the polarity orientation of microtubules within control and experimental dendrites, we used the standard "hooking" protocol. In this procedure, the cells are lysed in the presence of exogenous brain tubulin in a special microtubule assembly buffer that promotes the formation of lateral protofilament sheets on the existing microtubules. When viewed in cross section with the electron microscope, these sheets appear as hooked appendages on the microtu- bules. The curvature of the "hooks" reveals the orientation of the microtubule. A clockwise hook as viewed from the distal end of the process indicates that the microtubule is oriented with its plus end distal to the cell body, and a counterclockwise hook indicates the opposite. The procedure was performed, and the data were interpreted as previously described (Sharp et al., 1995, 1997b; Yu et al., 1997).

\section{RESULTS}

We have documented in a previous study that treatment with antisense oligonucleotides is an effective means to specifically and rapidly reduce levels of CHO1/MKLP1 protein within cultured rat sympathetic neurons (Sharp et al., 1997b). In these earlier studies, cultures were exposed to oligonucleotides before dendritic differentiation. We performed dose-response studies using two different antisense oligonucleotide sequences and their corresponding sense controls, and found both of the antisense sequences to be highly effective in diminishing protein levels in a dose-dependent and time-dependent fashion. There were no observed ill-effects on the health of the neurons, other than the fact that dendrites did not develop. No diminution in any other cytoskeletal proteins was observed with the antisense treatments. No diminution in CHO1/ MKLP1 or any other proteins were observed with the sense sequences, nor were any morphological changes observed. After rinsing out the antisense oligonucleotides, CHO1/MKLP1 expression resumed, as did the normal program of dendritic differentiation. Having already reported this set of experiments, we felt comfortable using one of the two antisense sequences for the present study, along with its corresponding sense sequence as a control. To assess the results of depleting CHO1/MKLP1 after dendrites had developed, cultures were exposed to the oligonucleotides on the fourteenth day after plating, by which time the vast majority of neurons had developed robust dendritic arbors. A concentration of $1 \mu \mathrm{M}$ was used because this concentration was shown in our earlier studies to be the minimum required to effectively suppress CHO1/MKLP1 expression, thus leading to a rapid depletion of the protein.

\section{Morphological changes in cultured neurons during antisense treatment}

We first wished to assess morphological changes that occur in the presence of the antisense oligonucleotides. Morphology was assessed either using phase-contrast optics or using fluorescence microscopy after immunostaining the cultures for a poorly phosphorylated epitope of neurofilament protein that is enriched in dendrites versus axons. We found that immunostaining with this antibody revealed both axons and dendrites, but that the dendrites were illuminated particularly well. Cultures that had not been treated with oligonucleotides and cultures treated with the sense oligonucleotides showed indistinguishable morphological characteristics, whereas cultures exposed to the antisense oligonucleotides were markedly different. In untreated cultures and cultures treated with sense oligonucleotides for 1,2 , or $3 \mathrm{~d}$, approximately half the neurons showed a very robust dendritic arbor (Figs. $1 a-c$, $2 a$ ). These cells showed at least three thick tapering curvaceous dendrites that were each $\sim 30$ - to $50-\mu \mathrm{m}$-long and at least $3-\mu \mathrm{m}$ wide at their base. Fewer than $10 \%$ of the neurons showed no thick tapering dendrites whatsoever, but instead consisted only of axonlike processes that only tapered over a few micrometers near the cell body if at all (Fig. 1d). The remaining neurons consisted of at least one thick tapering dendrite, but in general displayed a less robust dendritic morphology than the majority of the neurons. These morphological differences among individual cells in the control cultures may result from heterogeneity of neuronal types in the superior cervical ganglia or may represent the failure of a small number of the neurons to differentiate as robustly as they might have under these culture conditions. In the antisense cultures, at days 1,2 , and 3 , there were no neurons that displayed the full robust dendritic arbor displayed by most of the neurons in the untreated and sense-treated cultures (Figs. $2 b-h$ ). The majority of the neurons displayed no large thick tapering processes whatsoever and were similar in appearance to the small number of neurons in 

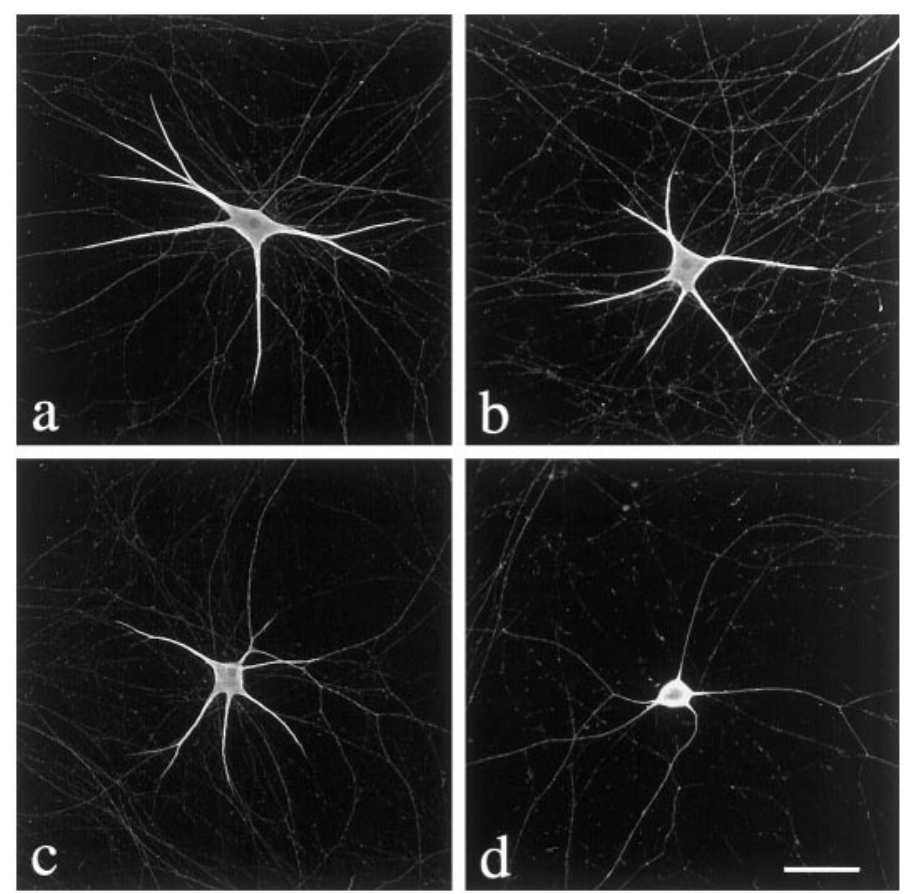

Figure 1. Morphology of cultured rat sympathetic neurons revealed by neurofilament immunostaining. Shown here are examples of the morphology of rat sympathetic neurons that were cultured in the presence of OP-1 to promote robust dendritic differentiation. After 2 weeks in culture, the vast majority of the neurons showed thick, tapering dendrites. Cultures were immunostained with an antibody to a poorly phosphorylated neurofilament epitope that is highly enriched in dendrites compared to axons. Most of the neurons showed three or more robust thick, tapering curvaceous dendrites that were at least $30-50 \mu \mathrm{m}$ in length. Some neurons showed a somewhat less robust dendritic arbor, but clearly showed unmistakable dendrites. Fewer than $10 \%$ of the neurons showed no robust dendrites $(d)$. Scale bar, $30 \mu \mathrm{m}$.

untreated and sense-treated cultures that displayed no robust dendrites (Fig. $2 h$ ). At $1 \mathrm{~d}$ of antisense treatment, $\sim 70 \%$ of the cells showed this "dendrite-less" morphology. This proportion progressively increased to $\sim 75$ and $80 \%$ at 2 and $3 \mathrm{~d}$ in antisense, respectively. Prolonged exposure to antisense (up to $12 \mathrm{~d}$ ) resulted in further loss of dendrites, but even after the longer exposure times, a small number of the cells $(<10 \%)$ continued to display processes with dendritic morphology (data not shown).

We focused most of our attention on the cultures treated with antisense for $1 \mathrm{~d}$, because the most dramatic changes in morphology occurred over the first $24 \mathrm{hr}$. In addition, we felt that the most informative cells to examine were those whose processes were in a transitional phase of losing their dendritic characteristics. As dendrites are lost from the cultures, do they gradually decay, do they retract into the cell body, or do they undergo transformation into processes with axonal characteristics? The "experimental" dendrites of these cells were typically different in appearance from any of the processes observed in the untreated or sense-treated cultures. The neuron shown in Figure $2 b$ displays experimental dendrites that are clearly longer than typical control dendrites, less tapered, but still curvaceous. Unlike the dendrites of this cell, the vast majority of dendrites in the antisense-treated cultures lost their curvaceous appearance and appeared much straighter and more taut. Figure $2 c$ shows a neuron with dendrites that are somewhat thinner and less tapered than control dendrites, and clearly less curvaceous. At least one of the dendrites is longer than typical controls. The remaining panels (panels $d-h$ ) show neurons with processes in more advanced stages of their loss of dendritic characteristics.

Even with the assistance of the neurofilament staining, it was still impossible to conclude whether the dendrites of the antisensetreated cells were shortening or whether they were becoming thin-
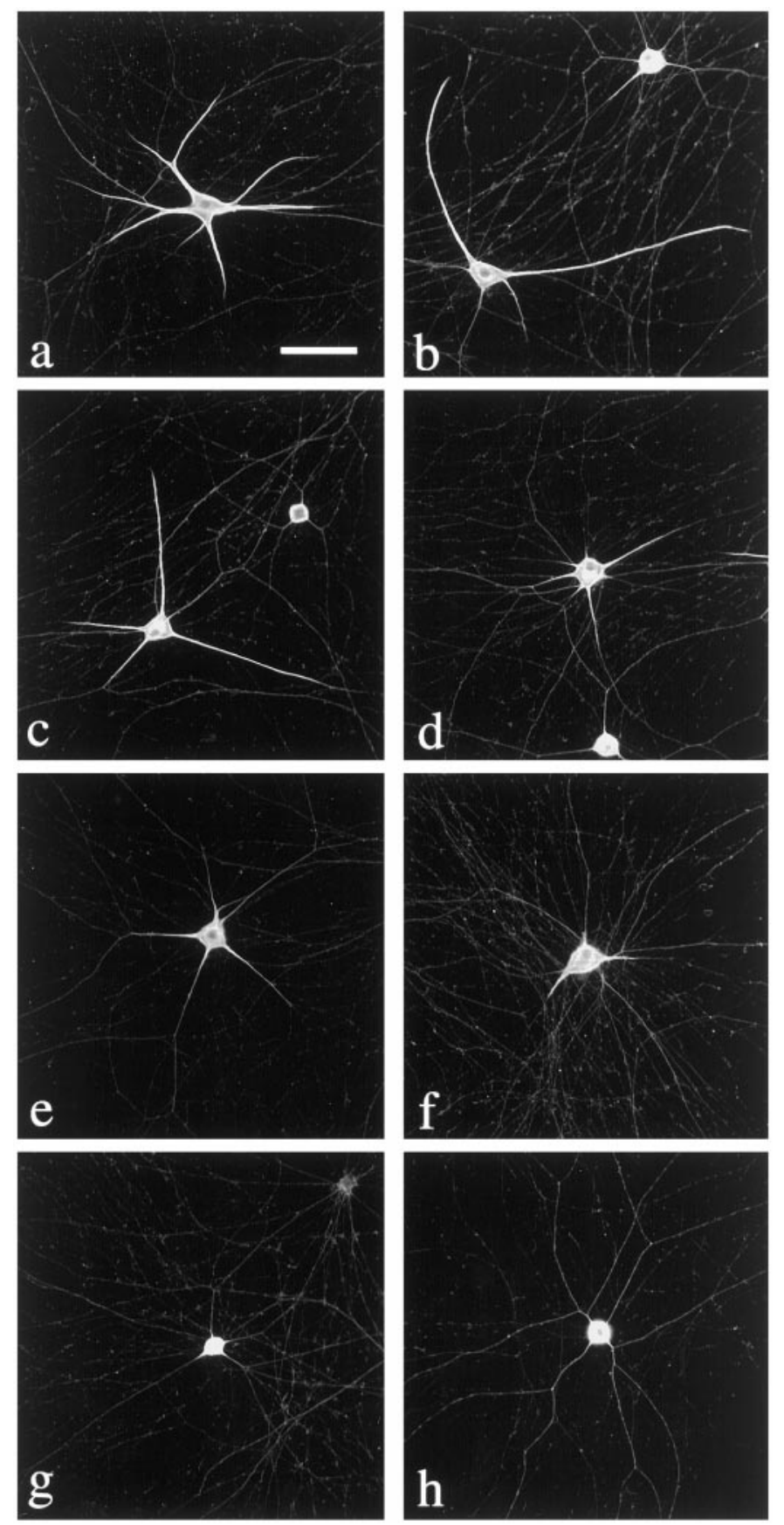

Figure 2. Morphology of cultured rat sympathetic neurons exposed to CHO1/MKLP1-antisense for $1 \mathrm{~d}$ revealed by neurofilament immunostaining. Shown here are neurons treated with sense or antisense oligonucleotides specific for CHO1/MKLP1. The neurons shown here were immunostained with the same neurofilament antibody as in Figure 1. $a$ shows a sense control with a robust dendritic arbor similar to that observed in typical control neurons. The remaining panels show antisense-treated neurons with clearly altered morphologies. The neuron shown in $b$ displays dendrites that are longer than typical control dendrites, less tapered but still curvaceous. The vast majority of the experimental dendrites lost their curvaceous appearance and appeared straight and taut. $c$ shows a neuron with dendrites that are somewhat thinner and less tapered than control dendrites, and less curvaceous. At least one of the dendrites is longer than typical controls. The remaining panels $(d-h)$ show neurons in more advanced stages of their loss of dendritic morphology. The tapered regions of the dendrites appear to wither in a distoproximal fashion, but the actual length of the processes is difficult to assess because of bundling with neighboring axons. Scale bar, $40 \mu \mathrm{m}$.

ner, losing their taper, and actually elongating. In cultures of rat sympathetic neurons, axons tend to bundle with dendrites, and hence it is difficult to discern the actual tip of a dendrite amid the neighboring axons that surround it. To resolve the issue, we micro- 

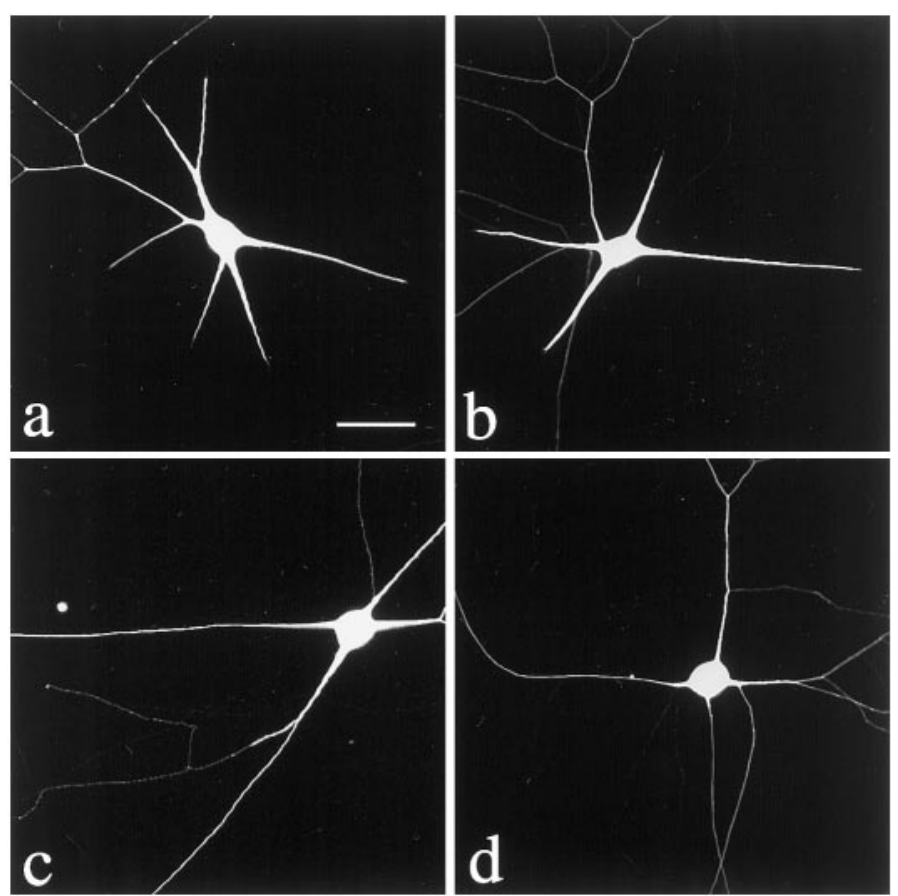

Figure 3. Morphology of individual neurons revealed by Lucifer yellow dye injections. Immunostains shown in Figures 1 and 2 suggest that during antisense treatment, the tapering regions of the dendrites wither, but it is unclear whether the entire length of the process increases or decreases because of the bundling of the dendrites with axons from neighboring cells. To investigate this issue, we injected Lucifer yellow into individual neurons to reveal their neuritic arbor. $a$ shows an untreated neuron, $b$ shows a sense-treated neuron, and $c$ and $d$ show antisense-treated neurons. Treatments were for $1 \mathrm{~d}$. In the untreated and sense-treated neurons, the tips of the dendrites are clearly distinguishable. Typical dendrites were 30-50 $\mu \mathrm{m}$ in length. In the antisense-treated neurons, the dendrites are substantially longer than controls. In many cases, the dendrites had elongated to well over $100 \mu \mathrm{m}$ over the first day in antisense. Scale bar, $30 \mu \mathrm{m}$.

injected a small number of cells in each culture with Lucifer yellow, a fluorescent dye that rapidly diff uses throughout the neuron (Higgins et al., 1991). A total of 50 cells under each experimental condition were examined. As shown in Figure 3, $a$ and $b$, the dye injections were very effective at revealing the tips of the dendrites in untreated and sense-treated neurons. Each dendrite was 30-50 $\mu \mathrm{m}$ in length. As shown in Figure 3,c and $d$, the dye injections revealed that the long slender axon-like regions that extended from the withering tapered regions of the experimental dendrites of the antisense-treated neurons were indeed directly continuous with these regions. We found that these elongated processes usually became too thin and too tortuous to identify their tips with confidence, but nevertheless, it was clear that their lengths typically exceeded $100 \mu \mathrm{m}$ and often exceeded hundreds of micrometers, even after the first day in antisense. Thus, during antisense treatment, the thick-tapering regions of the dendrites gradually withered toward the cell body, whereas the total length of the process underwent significant elongation.

\section{Reduction in CHO1/MKLP1 levels during antisense treatment}

As noted above, our previous study demonstrated the specificity and efficacy of the antisense treatment in terms of diminishing CHO1/MKLP1 levels in cultured rat sympathetic neurons. Such efficacy and specificity have also been shown in studies using the same CHO1/MKLP1 antisense sequence on cultured neuroblastoma cells (Yu et al., 1997) and cultured podocytes (Kobayashi et al., 1998). Nevertheless, we chose to perform quantitative immunofluorescence analyses on the levels of CHO1/MKLP1 remaining in neurons after treatment with the antisense to ascertain the degree to which the levels were reduced and also to investigate whether the levels of the protein correspond in any way to specific
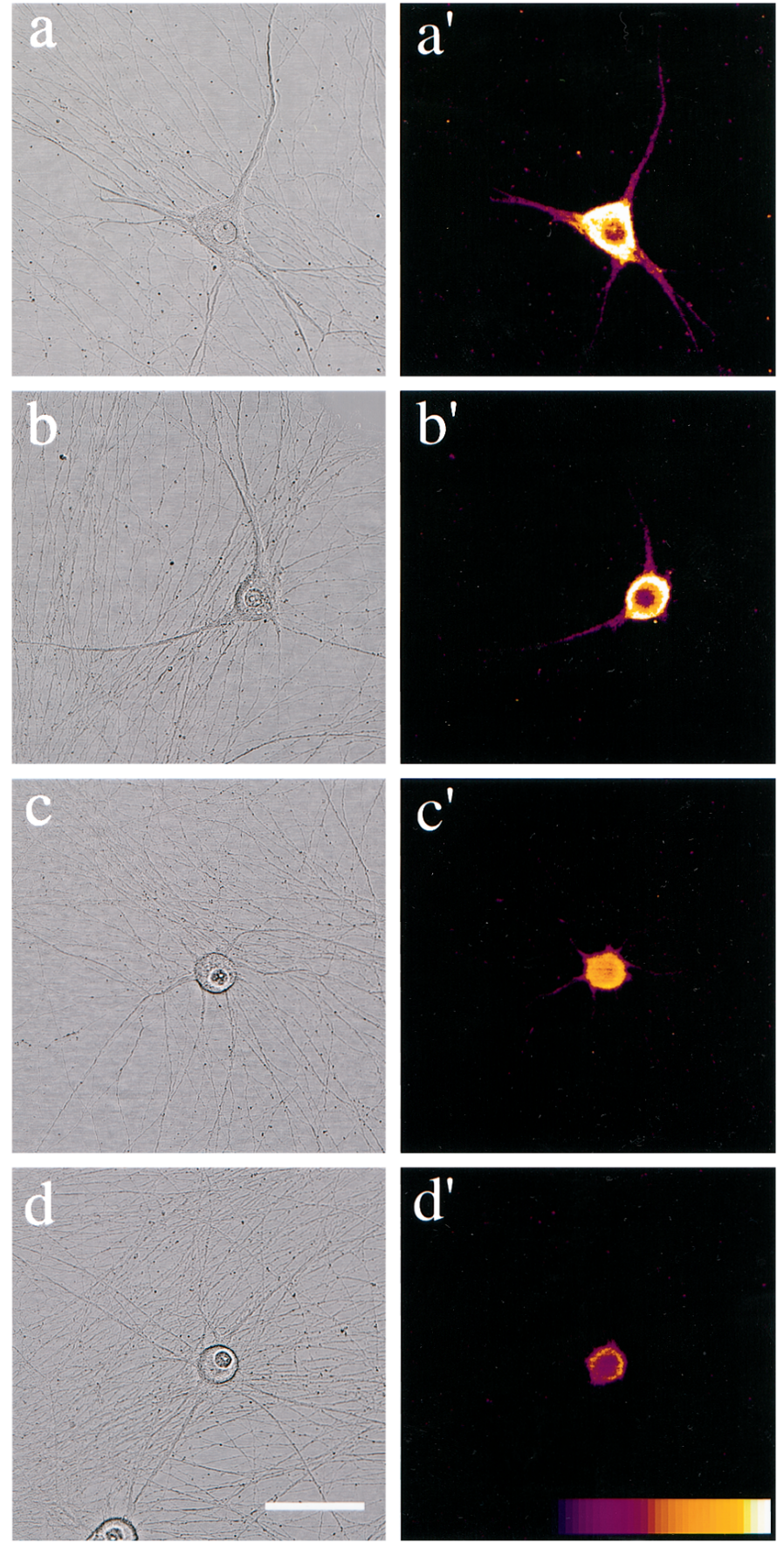

Figure 4. Immunofluorescence analyses on the levels of CHO1/MKLP1 in control and antisense-treated neurons. After $1 \mathrm{~d}$ in antisense, cultures were fixed in cold methanol, and CHO1/MKLP1 was visualized using immunofluorescence microscopy. DIC images of the fixed cells are shown in the left-hand column, whereas corresponding immunofluorescence images are shown in the right-hand column. $a$ and $a^{\prime}$ show an untreated neuron with robust dendrites and strong CHO1/MKLP1 immunoreactivity in the cell body and dendrites but not the axonal network. $b$ and $b^{\prime}$ show another untreated neuron with somewhat less robust dendrites. The immunoreactivity is correspondingly somewhat less intense. $c$ and $c^{\prime}$ show an antisensetreated neuron wherein the withered tapered regions of dendrites are still apparent. Immunoreactivity is significantly lower than in control neurons. $d$ and $d^{\prime}$ show an antisense-treated neuron wherein the dendrites have almost completely reverted to an axonal morphology. The immunoreactivity is even lower yet. Scale bar, $35 \mu \mathrm{m}$.

features of neuronal morphology. Fluorescence intensities were expressed in arbitrary fluorescence units (AFUs). Oligonucleotide treatments were for $1 \mathrm{~d}$. In 13 untreated and 15 sense-treated neurons with robust dendritic arbors, the CHO1/MKLP1 levels 

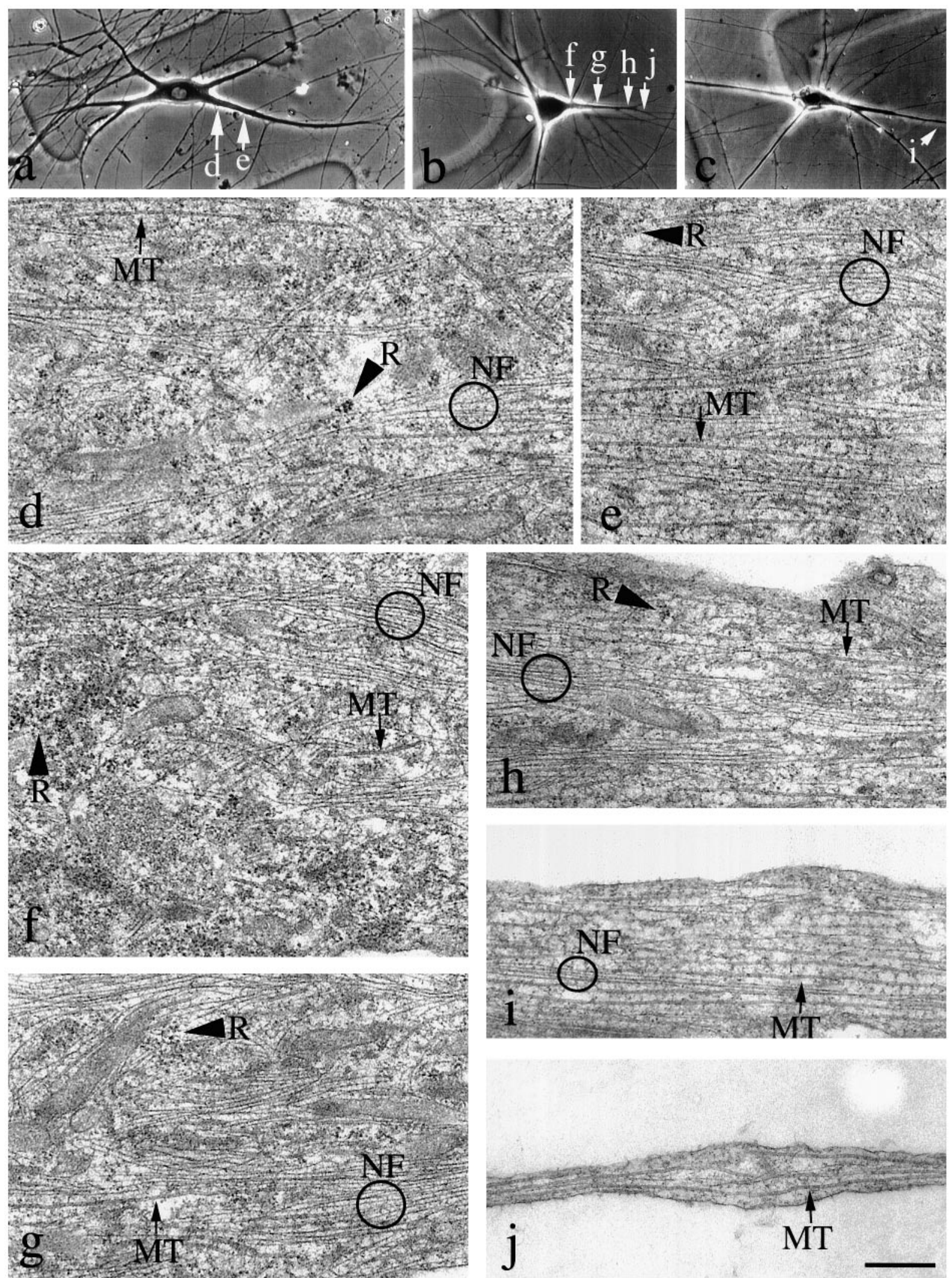

Figure 5. Electron microscopic analyses on dendrites of control and CHO1/MKLP1-antisense-treated neurons. Top panels show phase-contrast micrographs of embedded samples. $a$ is an untreated neuron, whereas $b$ and $c$ are antisense-treated. The remaining panels show electron micrographs from the indicated regions. $M T$, Microtubule; $N F$, neurofilament bundles typical of dendrites; $R$, ribosomes. Control neurons show abundant ribosomes near the cell body and fewer but still abundant levels farther down the length of the dendrite. Microtubules are scattered near the cell body, but more paraxial (but still not as paraxial as in axons) farther down the length of the dendrite. Neurofilament bundles are plentiful throughout. Ribosomes are still plentiful near the cell body of antisense-treated dendrites, but are dramatically diminished with distance, and virtually absent from the thinner distal regions of the original dendrite and the newly grown regions. Neurofilament bundles remain plentiful throughout most of the original length of the dendrite, but only sparse unbundled neurofilaments are present in the newly grown regions. Microtubules appear at relatively lower levels within the more proximal regions of the original dendrite, but at higher levels more distally. The microtubules appear to be more paraxial than in controls (Figure legend continues), 

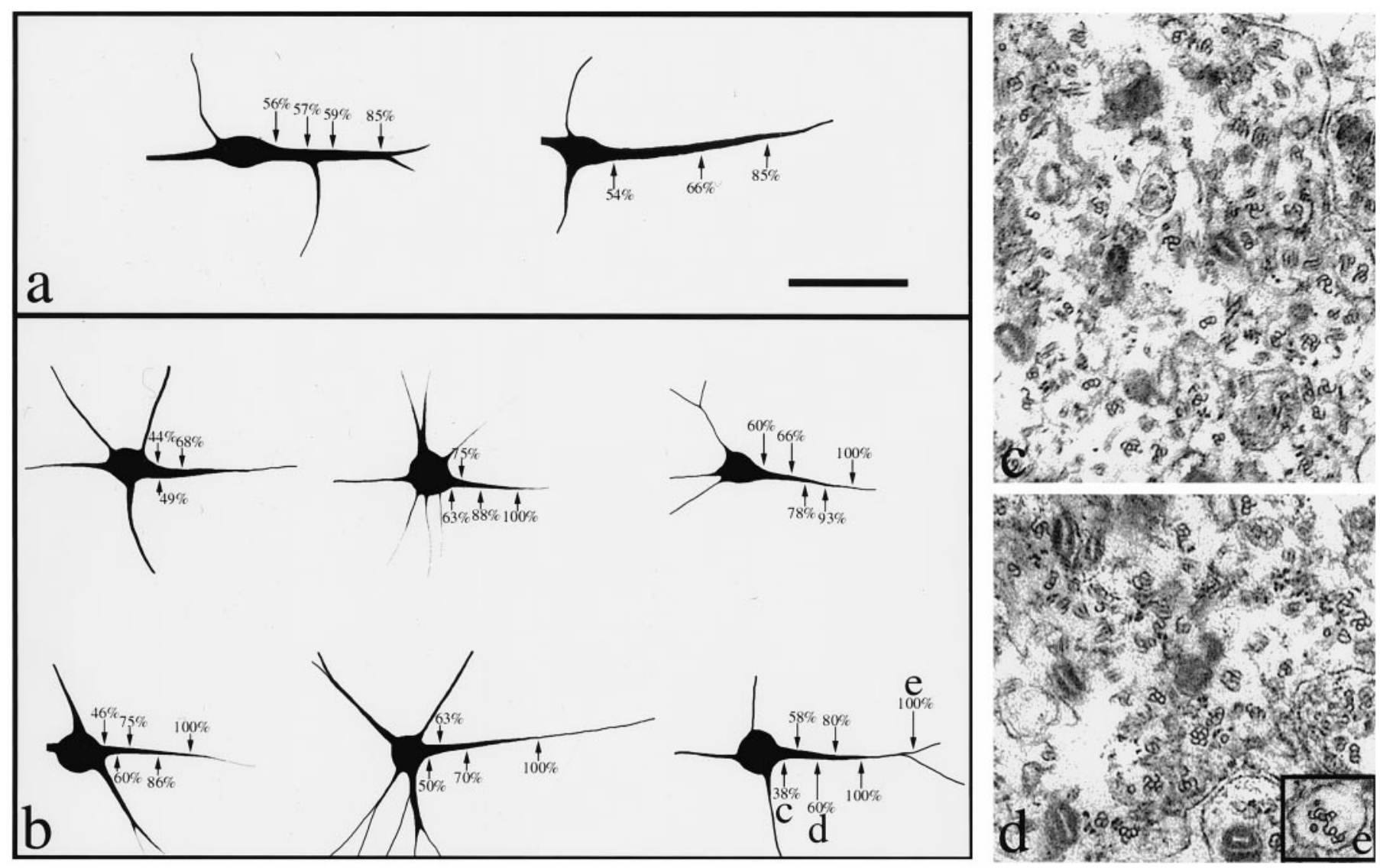

Figure 6. Microtubule polarity analyses on control and CHO1/MKLP1-antisense treated cultures. The standard "hooking" procedure was used to assess microtubule polarity orientation. Clockwise hooks indicate plus-end-distal microtubules, whereas counterclockwise hooks indicate minus-end-distal microtubules. $a$ shows the data from two control dendrites, whereas $b$ shows the data from six antisense-treated dendrites. The data are expressed as the percentages of clockwise hooks in different sampled regions. In control dendrites, slightly more than half of the hooks were clockwise in proximal and middle regions, with progressively higher percentages in the more distal regions. In dendrites that had completely thinned along their lengths, the percentage of clockwise hooks was $>95 \%$, indicating uniformly plus-end-distal microtubules (data not shown). The dendrites shown in $b$ are "transitional," in the sense that they still showed withering tapered regions. In all cases, the thinner distal regions showed predominantly or entirely clockwise hooks. In two of the six cases, the proportion of plus-end-distal microtubules was significantly higher than controls at corresponding sites throughout the length of the dendrite. In four of the six cases, this increase in the proportion of plus-end-distal microtubules was observed throughout most of the length of the dendrite, except in the most proximal region near the cell body, which actually showed a reduction in the proportion of clockwise hooks. Examples of the electron micrographs are shown in $c-e$, and the corresponding regions of the dendrite from which the electron micrographs were taken are shown by lettered-marked arrows in $b$. Scale bar: $a, b, 40 \mu \mathrm{m} ; c-e, 0.45 \mu \mathrm{m}$.

were all $>100$ AFUs $(151 \pm 30$ AFUs for untreated cultures and $130 \pm 21$ AFUs for sense-treated cultures). In seven untreated neurons and six sense-treated neurons with less robust dendrites, the CHO1/MKLP1 levels were all $>50$ AFHs but $<100$ AFUs $(83 \pm 12$ and $78 \pm 11$ AFUs, respectively). In nine untreated and nine sense-treated neurons with no dendrites, the levels were all $<60$ AFUs ( $49 \pm 9$ and $44 \pm 13$ AFUs, respectively). In 10 antisense-treated neurons with withering tapered regions, the levels were between 70 and 90 AFUs ( $75 \pm 9$ AFUs). In 10 antisensetreated neurons with no tapering regions remaining, the levels were all $<50$ AFUs $(40 \pm 11$ AFUs). These results, examples of which are shown in Figure 4, indicate that the antisense treatment (but not the sense treatment) was effective in reducing CHO1/MKLP1 levels (by an average of $\sim 40 \%$ across cells), and that the levels of this protein roughly correspond to the robustness of the dendritic arbor, both in antisense-treated and control neurons.

\section{Ultrastructural changes during antisense treatment}

To assess ultrastructural changes in dendrites that result from the antisense treatment, untreated and antisense-treated neurons were prepared for standard transmission electron microscopy. In control neurons (see Fig. $5 a$ for phase-contrast image), ribosomes were abundant throughout the length of the dendrite and were especially rich in the most proximal third of the process near the cell body (Fig. $5 d$ ). Microtubules were somewhat scattered near the cell body (Fig. $5 d$ ), but were more paraxial (but not as paraxial as in axons) farther down the length of the dendrite (Fig. 5e). Neurofilaments were plentiful throughout and formed dense bundles that were characteristic of dendrites but not axons (Baas et al., 1991). In the antisense-treated cultures that showed withering tapered regions (see Fig. 5, $b$ and $c$, for phase-contrast images), ribosomes were still plentiful near the cell body of antisense-treated dendrites (approximately the proximal fifth; Fig. $5 f$ ), but were dramatically diminished with distance and virtually absent from the thinner distal regions that still fell within the original length of the dendrite (Fig. $5 h$ ) and from the newly grown regions (Fig. 5i,j). Neurofilament bundles remain plentiful throughout most of the original length of the dendrite (Fig. $5 f-h$ ), but only sparse unbundled neurofilaments were present in the newly grown regions (Fig. 5i,j). Microtubules

$\leftarrow$

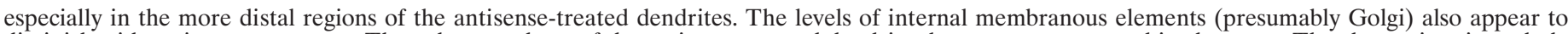

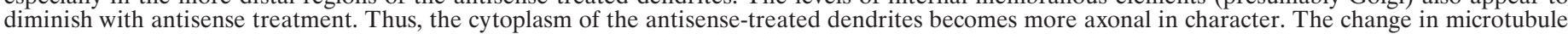

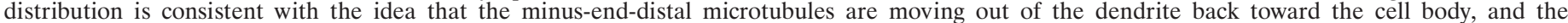
plus-end-distal microtubules are translocating anterogradely during antisense treatment. Scale bar, $0.5 \mu \mathrm{m}$. 

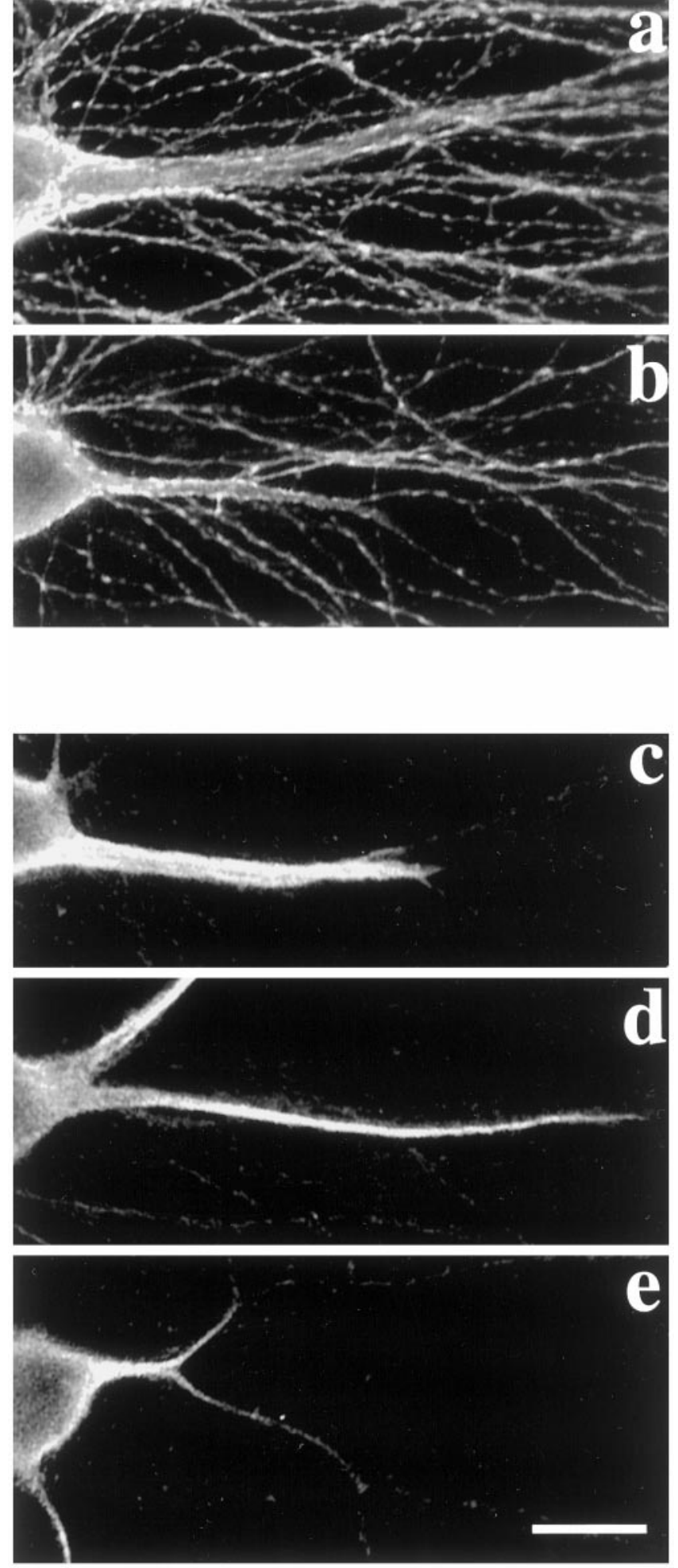

Figure 7. Tau-1 and MAP-2 immunofluorescence analyses on control and CHO1/MKLP1 antisense-treated neurons. $a$ and $b$ show an untreated and an antisense-treated neuron, both stained with the tau- 1 antibody. The dendrites of untreated cultures stained lightly and were clearly surrounded by more intensely-staining axons $(a)$. The withering regions of the antisense-treated dendrites showed no apparent increase in staining for tau- 1 and were still surrounded by more intensely stained axons $(b)$. $a$ shows an untreated neuron, whereas $d$ and $e$ show antisense-treated neurons, all stained for fulllength MAP-2. The dendrites of untreated cultures stained intensely for full-length MAP-2, whereas the axons showed little or no staining $(c)$. As the dendrites thinned and elongated in response to antisense-treatment, there was no immediate diminution in MAP-2 staining $(d)$, although the intense MAP-2 staining was gradually diminished as the thick tapering regions of the dendrites became progressively more withered $(e)$. Scale bar, $12 \mu \mathrm{m}$. appear at relatively lower levels within the more proximal regions of the original dendrite (Fig. $5 f$ ), but at higher levels more distally (Fig. $5 h-j)$. The microtubules appear to be more paraxial than in controls, especially in the more distal regions of the antisensetreated dendrites. The levels of internal membranous elements (presumably much of which is Golgi; Sharp et al., 1995) also appear to diminish with antisense treatment and with distance down the length of the antisense-treated dendrites. The cytoplasm of processes that had completely lost their taper was entirely similar to the cytoplasm within the thin distal regions of the "transitional" dendrites shown in Figure 5, $i$ and $j$ (data not shown). Thus, the cytoplasm of the dendrite becomes gradually more axonal in character during antisense treatment, and the organelles that are normally present in dendrites but not axons appear to retreat proximally toward the cell body as the dendrites lose their thickness and taper.

\section{Changes in microtubule polarity orientation as a result of CHO1/MKLP1 depletion}

The alterations in dendritic morphology and composition are consistent with a loss of minus-end-distal microtubules during $\mathrm{CHO} 1 /$ MKLP1 antisense treatment. To assess microtubule polarity orientation, we used the standard "hooking" procedure. In this method, cells are extracted in the presence of exogenous brain tubulin in a special buffer that promotes the addition of the exogenous tubulin onto the sides of pre-existing microtubules. The exogenous tubulin forms lateral curved appendages that appear as "hooks" when viewed in cross section electron microscopically. As viewed from the tip of the dendrite, clockwise hooks indicate plus-end-distal microtubules, whereas counterclockwise hooks indicate minus-end-distal microtubules. As previously reported, in control dendrites, slightly more than half of the microtubules were plus-end-distal in proximal and middle regions, with progressively higher percentages in the more distal regions (Baas et al., 1989, 1991). Data from two such control dendrites are shown in Figure $6 a$. We viewed $>20$ sections from dendrites that had completely thinned along their lengths and in all cases, the percentage of clockwise hooks was $>95 \%$, indicating uniformly plus-end-distal microtubules. Shown in Figure $6 b$ are the data from six examples of "transitional" dendrites that still showed withering tapered regions. In all cases, the thinner distal regions showed predominantly or entirely clockwise hooks. In two of the six cases, the proportion of plus-end-distal microtubules was significantly higher than controls at corresponding sites throughout the length of the dendrite. In four of the six cases, this increase in the proportion of plus-enddistal microtubules was observed throughout most of the length of the dendrite, except in the most proximal region near the cell body, which actually showed a reduction in the proportion of clockwise hooks (indicating an increase in the proportion of minus-end-distal microtubules). Examples of the electron micrographs are shown in Figure $6 c-e$. Taken together, these data are consistent with the view that minus-end-distal microtubules are gradually cleared from the antisense-treated dendrites and that microtubules of this orientation are chased back toward the cell body.

\section{Distribution of microtubule-associated proteins during antisense treatment}

Several studies have shown that the full-length isoform of MAP-2 is concentrated within dendrites, whereas a specific phosphorylated isoform of tau (recognized by the tau-1 antibody) is concentrated within the axon (Binder et al., 1985; Matus, 1994). The mechanisms responsible for this compartmentation and its functional significance remain unclear. In our hands, dendrites of untreated cultures stained lightly with the tau- 1 antibody and were clearly surrounded by more intensely staining axons (Fig. 7a). The axons in the culture varied in their level of intensity, perhaps as a function of their length or distance from the cell body. The withering regions of the antisense-treated dendrites showed no apparent increase in staining for tau-1 and were still surrounded by more intensely stained axons (Fig. 7b). Given the variability in the levels of axonal stain- 


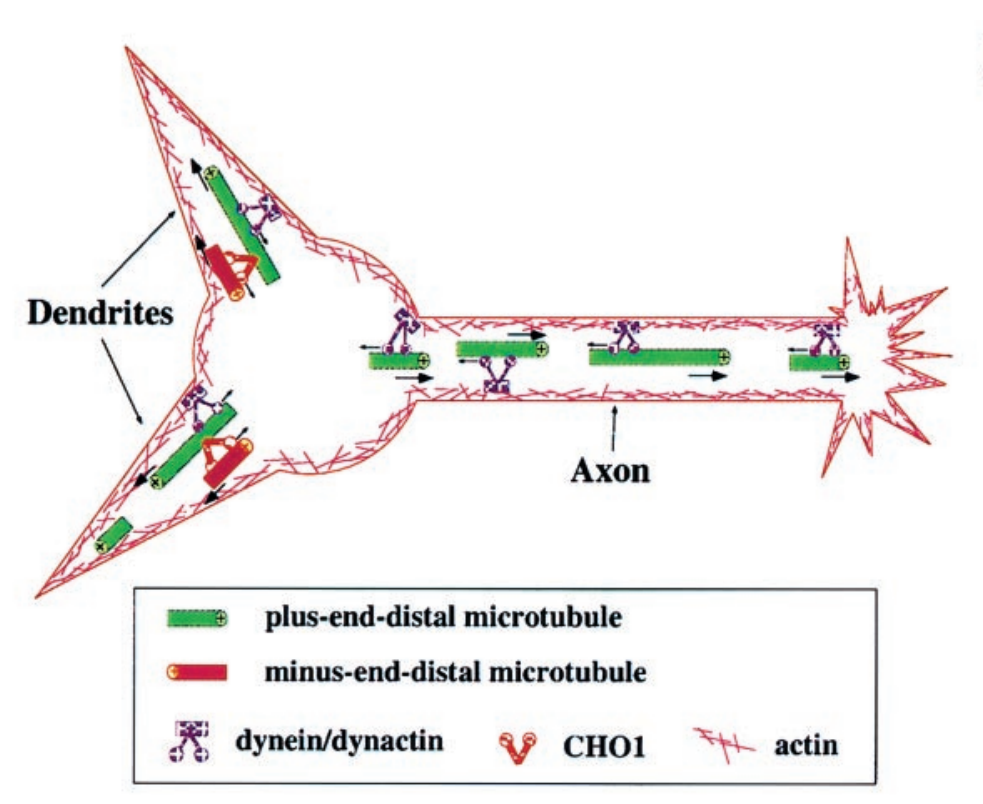

\section{$\mathbf{a}$}

\section{plus-end-distal microtubule}

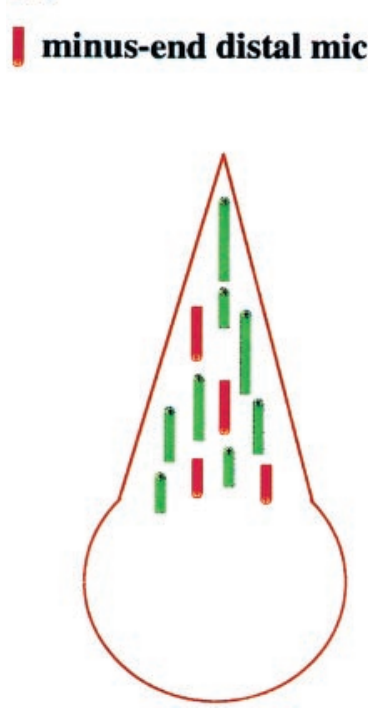

\section{tubule}
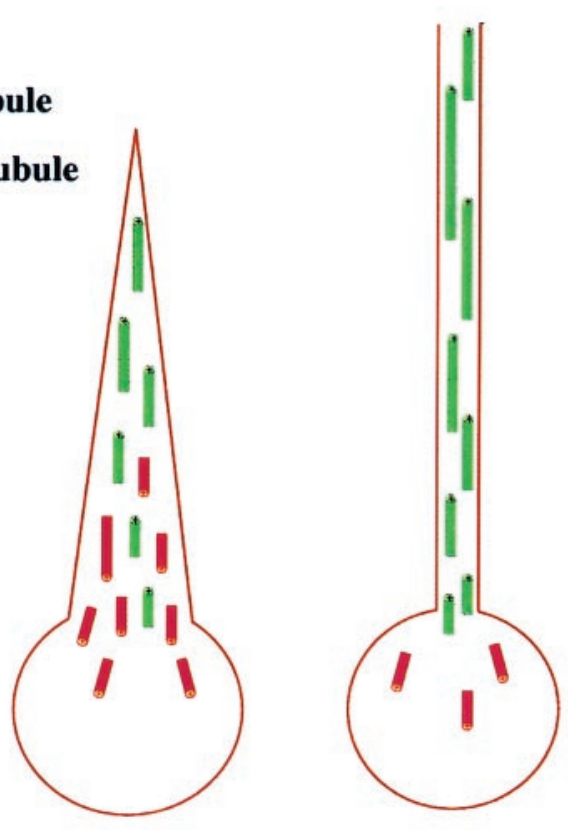

Figure 8. A model for the role of CHO1/MKLP1 in the dendrite. In our model $(a)$, cytoplasmic dynein transports microtubules with their plus ends leading (pushing against the actin cytomatrix) into the axon and the dendrites, whereas CHO1/MKLP1 transports microtubules anterogradely with their minus-ends-leading (pushing against the plus-end-distal microtubules) into the dendrites but not the axon. The forces generated by CHO1/MKLP1 create drag on the plus-end-distal microtubules, which impedes their dynein-driven transport. Both the suppression of dyneindriven microtubule transport and the nonuniform microtubule polarity pattern itself result in the characteristic morphological and compositional features that distinguish dendrites from the axon. This model makes certain predictions regarding what would happen if $\mathrm{CHO} 1 / \mathrm{MKLP} 1$ were depleted $(b)$. The model predicts that when $\mathrm{CHO} 1 /$ MKLP1 is depleted, the forces generated by cytoplasmic dynein should no longer be antagonized. The plus-enddistal microtubules should therefore be better able to move anterogradely, and the minus-end-distal microtubules should be transported (with their plus ends leading) back toward the cell body. As the minus-end-distal microtubules are cleared and the plus-end-distal microtubules move rapidly forward, the dendrite should gradually lose its taper, thin out, and elongate. These predictions are consistent with the redistribution of microtubules and the alterations in dendritic morphology observed in our depletion studies. Observed compositional changes are also consistent with the predictions of the model (data not shown in the schematic). ing, it was unclear whether the elongated distal regions of these dendrites stained any more or less intensely for tau compared to typical axons. The dendrites of untreated cultures stained intensely for full-length MAP-2, whereas the axons showed little or no staining (Fig. 7c). As the dendrites thinned and elongated, there was no immediate diminution in MAP-2 staining (Fig. $7 d$ ), although the intense MAP-2 staining was gradually diminished as the thick tapering regions of the dendrites withered (Fig. 7e). After the complete transformation of the dendrites to an axonal morphology, they were indistinguishable from the axons in the culture on the basis of their staining for these MAPs (data not shown). These studies show that a transformation to a more axonal MAP composition eventually occurs in the experimental dendrites, but that the signs of this occurring are not detectable as early as the signs of transformation to a more axonal morphology and ultrastructure.

\section{DISCUSSION}

In previous studies, it was established that CHO1/MKLP1 is enriched in dendrites but not axons and that inhibition of its expression before dendritic development prohibits dendrites from forming (Yu et al., 1997; Sharp et al., 1997b). On the basis of these observations, we tentatively concluded that CHO1/MKLP1 is essential for the transport of minus-end-distal microtubules into nascent dendrites and that the presence of the minus-end-distal microtubules is requisite for the acquisition of a dendritic morphology and organelle composition. Of course, there are alternative explanations for the manner by which a motor protein might be essential for dendritic differentiation, and it is important to note that the issue of microtubule transport has been controversial (for discussion, see Baas, 2000). In the present study, we have suppressed $\mathrm{CHO} 1 / \mathrm{MKLP} 1$ expression after dendrites had already formed and observed the results of a gradual depletion of the protein. If CHO1/MKLP1 functions as we have proposed, we can make predictions about how microtubules should redistribute, how organelles should redistribute, and how the morphology of the dendrite should change as the levels of CHO1/MKLP1 are diminished. Our model for the establishment of the neuronal microtubule arrays, shown schematically in Figure $8 a$, is based on the manner by which CHO1/MKLP1 and cytoplasmic dynein trans- 
port microtubules in vitro and in the mitotic spindle (for discussion, see Baas, 1999). In this model, cytoplasmic dynein transports microtubules into axons and dendrites with their plus ends leading by pushing against the actin cytomatrix (Ahmad et al., 1998), whereas CHO1/MKLP1 transports microtubules with their minus ends leading only into dendrites by pushing against the plus-enddistal microtubules (Sharp et al., 1997b). Once CHO1/MKLP1 is sufficiently depleted, the unopposed dynein-driven forces should redistribute the microtubules by transporting them all with their plus ends leading. This would drive the minus-end-distal microtubules back into the cell body, and the plus-end-distal microtubules would now move more efficiently forward. As the microtubule polarity pattern becomes gradually more plus-end-distal, the morphology and cytoplasmic composition of the dendrites should become gradually more axon-like.

The results of our studies bear out these predictions (Fig. 8b). As the levels of CHO1/MKLP1 are diminished, there is a dramatic redistribution of microtubules. Minus-end-distal microtubules gradually vacate the dendrite until the dendrite completely reverts to a uniformly plus-end-distal pattern of microtubule organization. During this transition, we documented cases in which there were abnormally high percentages of minus-end-distal microtubules in the proximal region of the dendrite at the same time as the proportion of plus-end-distal microtubules increased in the middle and more distal regions. These results are not consistent with the idea that the minus-end-distal microtubules simply vanish or depolymerize from the dendrite, but are entirely consistent with the predictions of our model, namely that these microtubules are chased back to the cell body by dynein-driven transport. In addition, these observations provide a new line of evidence favoring the view that tubulin is actively transported within neurons in the form of polymers (Baas, 2000). As this redistribution of microtubules occurred, there was a dramatic alteration in the morphology of the dendrite; the region still containing minus-end-distal microtubules continued to show taper until most or all of the microtubules of this orientation were depleted. The region cleared of minus-end-distal microtubules became thinner, elongated dramatically, and took on the morphological appearance of an axon. Eventually, the entire dendrite acquired an axonal morphology as the microtubule polarity pattern became uniformly plus-end-distal.

We suspect that there are two reasons for the morphological changes. The first reason relates directly to the forces generated by the motor. Once the CHO1/MKLP1-driven forces are diminished, a "drag" is removed on the anterograde transport of the plus-enddistal microtubules, thus permitting these microtubules to move as rapidly in dendrites as they do in the axon. This would promote rapid growth of the process. A shift in these forces might also explain why the experimental dendrites become less curvaceous and more taut. We have recently shown that tension in the axon results from myosin-driven forces on the microfilament array and that these forces are attenuated by the dynein-driven forces between the microtubule and the microfilament arrays (Ahmad et al., 2000). The CHO1/MKLP1-driven forces would be additive to the dynein-driven forces, thus further reducing the tension in the dendrite. Less overall tension would result in a less taut and more curvaceous process. Under conditions of depleted CHO1/MKLP1, we would expect tension in the dendrite to increase, thus producing a more taut and less curvaceous process. The other explanation for the morphological changes relates to the transport of membranous vesicles needed for the growth of the processes at their tips. Assuming that these vesicles move toward plus ends of microtubules, a uniformly plus-end-distal microtubule array would provide a unidirectional vector for the vesicles to move to the distal tip of the process. Thus, the process would be disposed to grow longer. A nonuniformly oriented microtubule array would not provide such a vector, and hence the vesicles would probably add to the dendrite along its sides, which would promote a thickening over an elongation of the process.

Compositional features of the dendrite also become gradually more axonal as CHO1/MKLP1 is depleted. Ribosomes and dendrite-enriched membranous structures (presumably Golgi elements) vacated the dendrite in a similar distoproximal manner as the minus-end-distal microtubules. In our model, the minus-enddistal microtubules provide a substrate for the transport of these organelles into the dendrite (Baas et al., 1988). In the absence of microtubules of this orientation, we would expect these organelles to move toward the minus ends of the plus-end-distal microtubules back toward the cell body. These observations support our hypothesis that these organelles are distributed on the basis of their transport toward minus-ends of microtubules. However, it should be noted that there are still many unresolved issues regarding exactly how such traffic is regulated. For example, for these organelles to accumulate within dendrites, it seems likely that there would have to be selective retention mechanisms in addition to selective transport mechanisms (Black and Baas, 1989). The present observations indicate that, if such mechanisms exist, they can be overwhelmed by the motor-driven forces that would transport these organelles retrogradely if not for the presence of the minus-end-distal microtubules.

Not all features of dendritic identity are so easy to understand on the basis of microtubule polarity orientation. The neurofilaments offer an interesting puzzle because they appear in both axons and dendrites, but differ in their phosphorylation state and bundling patterns in each type of process. Perhaps specific kinases or other regulators of neurofilament organization are mediated by features of microtubule organization, but how such a mechanism might be orchestrated remains unclear. The mechanisms by which MAP-2 and tau- 1 isoforms are compartmentalized also remain mysterious. These proteins alter their distribution as a result of $\mathrm{CHO} 1 /$ MKLP1 depletion, but this occurs more slowly than the morphological changes that we have documented. Interestingly, a plus-enddirected kinesin has recently been discovered which appears to be targeted for dendrites but not axons (Marszalek et al., 1999), and the mechanism for this targeting is also unclear. We find it compelling to contemplate that perhaps the acquisition of nonuniform microtubule polarity orientation could set into motion a cascade of events that leads to all of these various features of dendritic identity. Although this hypothesis will require more testing, initial support for it is provided by our studies showing that with sufficient time, depletion of CHO1/MKLP1 causes dendrites to lose their identity and literally "become axons" by all of the criteria that we have explored.

Our quantitative immunofluorescence studies suggest that neurons might normally regulate dendritic morphology by modulating the levels of CHO1/MKLP1. The control neurons with more robust dendritic arbors consistently showed stronger immunoreactivity. Treatment with the antisense drastically reduced the levels of the protein in most neurons, but the degree to which the protein was diminished varied from cell to cell. Neurons with less of the protein showed more significant loss of dendritic characteristics, which supports the idea that the robustness of the dendritic arbor could be regulated by the absolute amount of this protein. The antisense results also suggest that neurons might regulate $\mathrm{CHO} 1 /$ MKLP1 through changes in its half-life. After $1 \mathrm{~d}$ in antisense, the remaining CHO1/MKLP1 protein diminished more slowly, suggesting that there may be a more long-lived pool of the protein as well as a more short-lived pool. The fact that a small number of cells maintained CHO1/MKLP1 immunoreactivity and fairly robust dendrites even after $12 \mathrm{~d}$ in antisense suggests that the proportion of protein with a longer half-life may increase during neuronal maturation or vary significantly from cell to cell. Our previous in situ hybridization analyses showed that the levels of CHO1/MKLP1 messenger RNA are significantly lower in adult neurons (Ferhat et al., 1998), which would be consistent with a longer half-life of the available protein. Another possibility is that smaller amounts of CHO1/MKLP1 are needed later in development because more mature neurons use other proteins such as MAP-2 to retain the minus-end-distal microtubules through structural cross-links. These and other issues will require further study. 


\section{REFERENCES}

Ahmad FJ, Echeverri CJ, Vallee RB, Baas PW (1998) Cytoplasmic dynein and dynactin are required for the transport of microtubules into the axon. J Cell Biol 140:246-256.

Ahmad FJ, Hughey J, Wittmann T, Hyman A, Greaser M, Baas PW (2000) Motor proteins regulate force interactions between microtubules and microfilaments in the axon. Nat Cell Biol 2:276-280.

Baas PW (1999) Microtubules and neuronal polarity: lessons from mitosis. Neuron 22:23-31.

Baas PW (2000) Strategies for studying microtubule transport in the neuron. Microsc Res Tech 48:75-84.

Baas PW, Ahmad FJ (1993) The transport properties of axonal microtubules establish their polarity orientation. J Cell Biol 120:1427-1437.

Baas PW, Yu W (1996) A composite model for establishing the microtubule arrays of the neuron. Mol Neurobiol 12:145-161.

Baas PW, Deitch JS, Black MM, Banker GA (1988) Polarity orientation of microtubules in hippocampal neurons: uniformity in the axon and nonuniformity in the dendrite. Proc Natl Acad Sci USA 85:8335-8339.

Baas PW, Black MM, Banker GA (1989) Changes in microtubule polarity orientation during the development of hippocampal neurons in culture. J Cell Biol 109:3085-3094.

Baas PW, Slaughter T, Brown A, Black MM (1991) Microtubule dynamics in axons and dendrites. J Neurosci Res 30:134-153.

Binder LI, Frankfurter A, Rebhun LI (1985) The distribution of tau in the mammalian nervous system. J Cell Biol 101:1371-1378.

Black MM, Baas PW (1989) The basis of polarity in the neuron. Trends Neurosci 12:211-214.

Ferhat L, Kuriyama R, Lyons GE, Micales B, Baas PW (1998) Expression of the mitotic motor protein CHO1/MKLP1 in postmitotic neurons. Eur J Neurosci 10:1383-1393.

Higgins D, Lein PJ, Osterhout DJ, Johnson MI (1991) Tissue culture of mammalian autonomic neurons. In: Culturing nerve cells (Banker G, Goslin K, eds). Cambridge, MA: MIT.

Kobayashi N, Reiser J, Kriz W, Kuriyama R, Mundel P (1998) Nonuniform microtubular polarity established by CHO1/MKLP1 motor protein is necessary for process formation of podocytes. J Cell Biol 143:1961-1970.

Kuriyama R, Dragas-Granoic S, Maekawa T, Vassilev A, Khodjakov A, Kobayashi H (1994) Heterogeneity and microtubule interaction of the CHO1 antigen, a mitosis-specific kinesin-like protein. Analysis of subdomains expressed in insect Sf9 cells. J Cell Sci 107:3485-3499.

Marszalek JR, Weiner JA, Farlow SJ, Chun J, Goldstein LSB (1999) Novel dendritic kinesin sorting identified by different process targeting of two related kinesins: KIF21A and KIF21B J Cell Biol 145:469-479.

Matus A (1994) MAP2 in Microtubules (Hyams JS, Lloyd CW, eds), pp 156-166. New York: Wiley-Liss.

Nislow C, Lombillo VA, Kuriyama R, McIntosh JR (1992) A plus-enddirected motor that moves anti-parallel microtubules in vitro localizes to the interzone of mitotic spindles. Nature (Lond) 359:543-547.

Sharp DJ, Yu W, Baas PW (1995) Transport of dendritic microtubules establishes their nonuniform polarity orientation. J Cell Biol 130:93-104.

Sharp DJ, Kuriyama R, Baas PW (1996) Expression of a kinesinrelated motor protein in Sf9 cells induces them to extend dendrite-like processes with nonuniform microtubule polarity orientation. J Neurosci 16:4370-4375.

Sharp DJ, Kuriyama R, Baas PW (1997a) Expression of a minus-enddirected motor protein induces Sf9 cells to form axon-like processes with uniform microtubule polarity orientation. J Cell Sci 110:2373-2380.

Sharp DJ, Yu W, Ferhat L, Kuriyama R, Rueger DC, Baas PW (1997b) Identification of a motor protein essential for dendritic differentiation. J Cell Biol 138:833-843.

Sellitto C, Kuriyama R (1988) Distribution of a matrix component of the midbody during the cell cycle in Chinese hamster ovary cells. J Cell Biol 106:531-439.

Yu W, Baas PW (1995) The growth of the axon is not dependent upon net microtubule assembly at its distal tip. J Neurosci 15:6827-6833.

Yu W, Sharp DJ, Kuriyama R, Mallik P, Baas PW (1997) Inhibition of a mitotic motor protein compromises the formation of dendrite-like processes from neuroblastoma cells. J Cell Biol 136:659-668. 